\title{
Beamforming Array for Detecting Buried Land Mines
}

\author{
Seung-Ho Lee and Waymond R. Scott, Jr. \\ School of Electrical and Computer Engineering \\ Georgia Institute of Technology \\ Atlanta, GA 30332-0250
}

\begin{abstract}
A beamforming array is investigated for use in a radar system that is part of a hybrid acoustic/electromagnetic technique for detecting land mines. The radar is used to measure the surface displacement of the earth due to acoustic waves in the earth. The beamforming array is used to obtain small spatial resolution for the measurement of the displacement while allowing an adequate standoff distance for the radar. The tradeoffs between the resolution and the sidelobes of the array are investigated. Finite-difference time-domain and experimental models have been implemented to examine the feasibility of the beamforming array.
\end{abstract}

Keywords: Beamforming Array, Mine Detection, Displacement Measurement, FDTD

\section{INTRODUCTION}

This work is part of a project in which a land mine detection system is being studied that simultaneously uses both acoustic and electromagnetic waves. ${ }^{1}$ The system consists of an electromagnetic radar and an acoustic source. An acoustic wave is launched into the earth, and the acoustic wave causes the surface of the earth and the mine to be displaced. The radar is used to measure the displacements and, thus, detect the mine. Currently the radar uses an open-ended waveguide for the antenna. However, this antenna must be placed within a few centimeters of the surface of the earth in order to get sufficiently small spatial resolution. In this work, we investigate using a beamforming array so that the antenna can be placed farther from the surface while maintaining sufficiently small spatial resolution. The array can be implemented either by constructing an array of N-elements and recording the signal from all of the elements simultaneously or by using a single element that is scanned to construct a synthetic array. The most practical solution will be to probably use a combination of these techniques and scan a subset of the N-element array to more quickly construct the synthetic array.

A simple analytical model is developed for the beamforming array. This model is used to investigate the tradeoffs between the resolution and the sidelobes of the array as a function of the height of the array and the method of focusing used in the beamforming. A finite-difference time-domain model (FDTD) and an experimental model have been implemented to examine the feasibility of the beamforming array. The results from these models are promising. The array is shown to have sufficient resolution for the mine detection technique mentioned above.

\section{BEAMFORMING ARRAY}

Figure 1 is a schematic diagram of an N-element beamforming array. The elements are placed on a plane parallel to the x-y plane, assumed to be identical, and located at the positions $\vec{R}_{i}^{\prime}$. Assuming an isotropic source is located at the position $\vec{R}$, the voltage received by the $i^{t h}$ element can be expressed as

$$
V_{i}(\vec{R})=C \frac{\cos \psi_{i}}{\left|\vec{R}-\vec{R}_{i}^{\prime}\right|} e^{-j k\left|\vec{R}-\vec{R}_{i}^{\prime}\right|}
$$

where $\mathrm{C}$ is a constant. Here, the electric field is treated as a scalar, and the elements are assumed to have the simple angular dependence $\cos \psi_{i}$, where $\psi_{i}$ is the angle between the propagation direction and the z-direction.

The terminal voltages of the elements of the array are summed with the appropriate weighting function to focus the array at the position $\overrightarrow{R_{f}}$. The phase of the weighting function is chosen so that the voltages will add constructively

E-mail and Tel.: S.H. Lee, gt2332b@prism.gatech.edu, 404-894-3123, W. R. Scott, Jr. : wrscott@ee.gatech.edu, 404-894-3084 


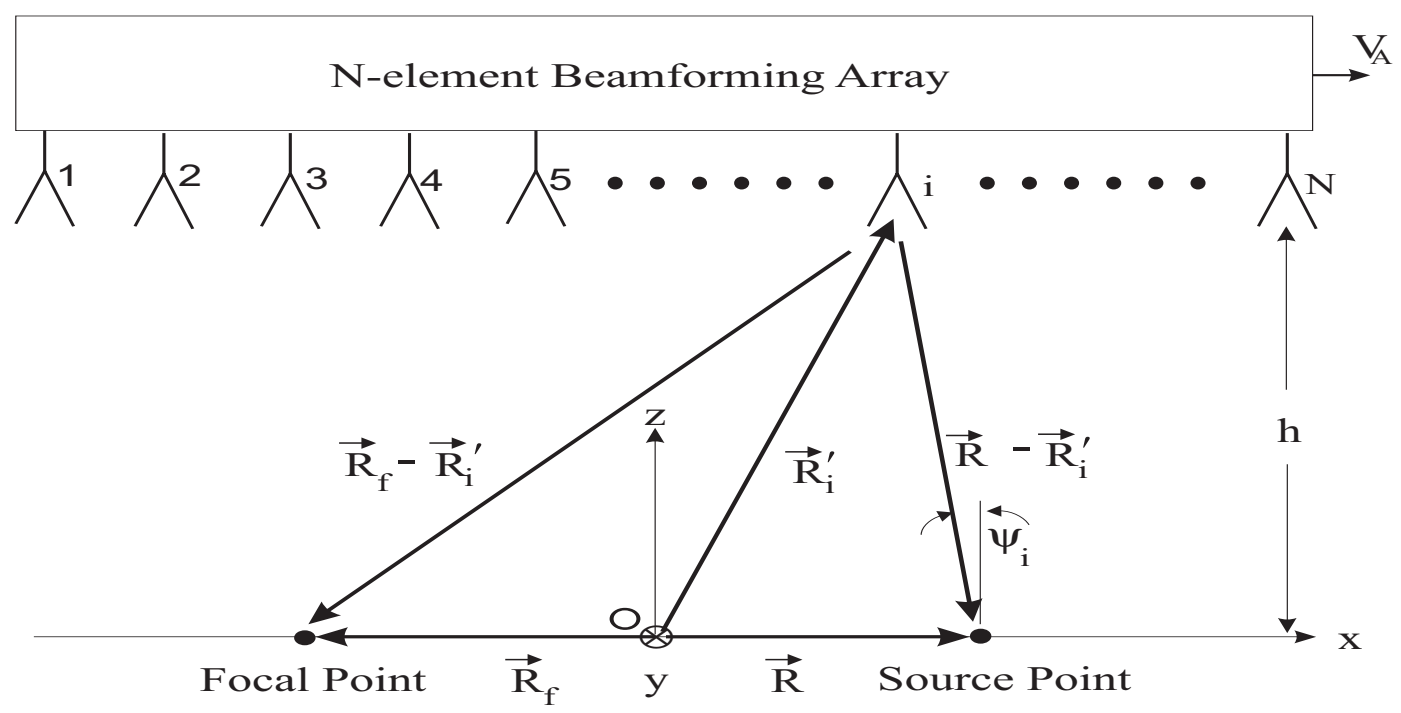

Figure 1. Beamforming array configuration

when the source is at the position $\overrightarrow{R_{f}}$, and the amplitude of the weighting function is chosen to control the sidelobe levels of the array. The output of the array is then

$$
V_{A}\left(\vec{R}, \overrightarrow{R_{f}}\right)=\sum_{i=1}^{N} V_{i}(\vec{R}) W\left(\overrightarrow{R_{i}^{\prime}}, \overrightarrow{R_{f}}\right) e^{j k\left|\overrightarrow{R_{f}}-\overrightarrow{R_{i}^{\prime}}\right|}
$$

where $W$ is a window function used to adjust the amplitude of the weighting function. We have determined empirically that a Gaussian window function performs well:

$$
W\left(\overrightarrow{R_{i}^{\prime}}, \overrightarrow{R_{f}}\right)=\exp \left(-\frac{1}{2 \tau^{2}}\left|\hat{z} \times\left(\overrightarrow{R_{f}}-\overrightarrow{R_{i}^{\prime}}\right)\right|^{2}\right),
$$

where $\tau$ is a parameter used to adjust the width of the window function. The window function is maximum directly above $\overrightarrow{R_{f}},\left|\hat{z} \times\left(\overrightarrow{R_{i}^{\prime}}-\overrightarrow{R_{f}}\right)\right|=0$, and it decreases with increasing distances from this point.

A graph of the output of the array as a function of the source position for several values of $\tau$ is shown in Fig.2. The array consists of 59 by 59 identical elements equally spaced on a square grid; they are spaced $0.47 \lambda$ apart. The array is centered on the point $(x=0, y=0, z=h=5.33 \lambda)$. The isotropic source is scanned from $x=-10 \lambda$ to $x=10 \lambda$ with $y=0$ and $z=0$. The array is focused at $x=y=z=0$. Since the beamforming array is focused at the center of the scan, the maximum output is obtained when the isotropic source is located at the center of the scan as expected. The sidelobe levels are seen to be quite high, and the beamwidth is seen to be very narrow when the window is very broad $(\tau=12.0 \lambda)$. As $\tau$ decreases, the sidelobe levels drop at the expense of wider beamwidths.

In Fig.3(b), the 3dB beamwidth and the first sidelobe level are plotted as a function of $\tau$ for three different values of $h$. The beamwidth is seen to decrease when $\tau$ is increased and when $h$ is decreased. However, the first sidelobe level behaves in an opposite manner; the first sidelobe level is seen to increase when $\tau$ is increased and when $h$ is decreased. Figure 3(a) is a similar graph for a 2-D case. Here, the elements and the source are assumed to have an infinite length in the y-direction. In this configuration, the beamforming array consists of 59 identical elements along the $\mathrm{x}$-direction only. The results for the $2-\mathrm{D}$ case are very close to those for the 3 -D case.

Figure 4 shows the output of the array with $\tau=2.67 \lambda$ as a function of the $\mathrm{x}$ - and $\mathrm{z}$-coordinates of the isotropic source. The configuration of the beamforming array is the same as the above 3 -D case with $h=5.33 \lambda$. The array is seen to be focused at the origin; the beamwidth is narrowest along the line $z=0$. However, note that the beamwidth does not change significantly when $z$ is varied in a range of approximately, $-\lambda<z<\lambda$. 


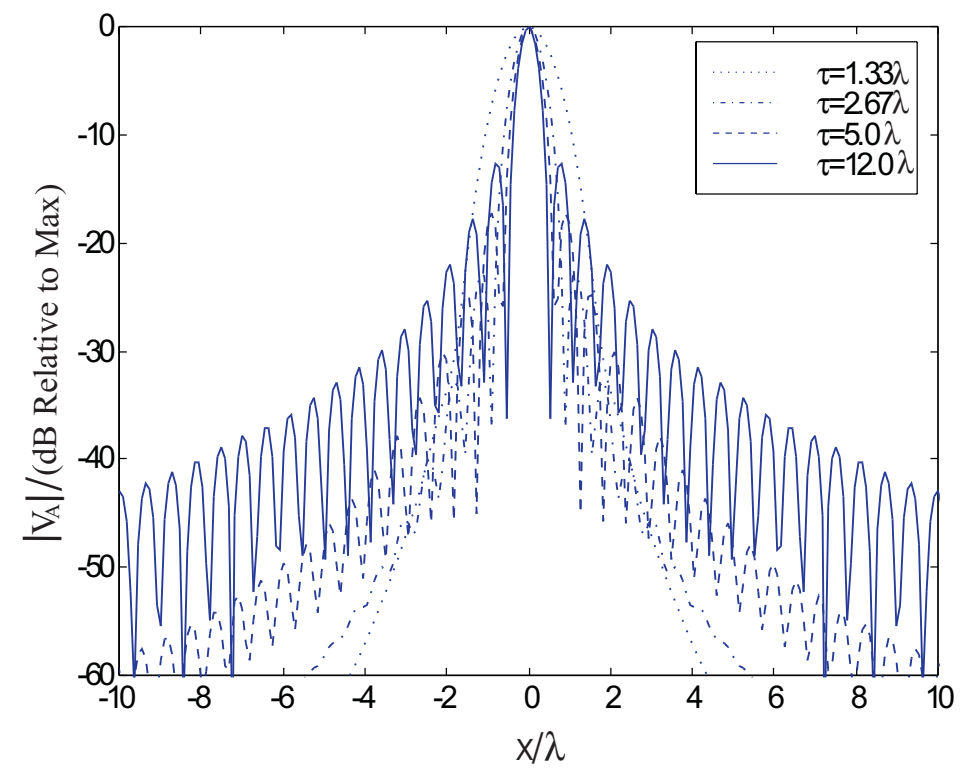

Figure 2. The output of the array as a function of the source position for different values of $\tau$ when $\mathrm{h}=5.33 \lambda$

\section{FDTD MODEL}

A two-dimensional FDTD model has been implemented to study the beamforming array. A diagram of the model is shown in Fig.5. A total field/scattered field formulation was used to inject an electromagnetic plane wave, an uniaxial perfectly matched layer ${ }^{2}$ was used to truncate the boundaries, and the earth was modeled as a lossy half space. The surface of the earth is displaced due to an acoustic wave traveling in the earth. These displacements are included in the model. The electromagnetic plane wave is reflected from the boundary between the air and the earth, and the total field (both incident and reflected waves) is recorded at the observation points. The reflected wave is obtained by subtracting the incident wave from the total field; the incident wave is calculated in the implementation of the total field/scattered field interface. ${ }^{3}$ The reflected electromagnetic waves are recorded both with and without the surface displacements.

The acoustic wave travels much slower than the electromagnetic wave. Thus, it is impractical to run the FDTD simulation for the time it would take the acoustic wave to travel across the model, because of the number of time steps that would be required. Fortunately, this is not necessary. In a given period of time, the acoustic wave travels $10^{-6}$ times as far as does the electromagnetic wave. The acoustic wave will also travel a very short distance with respect to its width during the total travel time of the electromagnetic wave. Thus, it is possible to decouple the time scales for the acoustic and the electromagnetic waves. The electromagnetic simulation is run with the acoustic wave at discrete positions on the boundary.

The surface displacements would seem to be very difficult to model in the FDTD method because they are very small compared to the size of a FDTD cell. However, it has been shown that displacements as small as $10^{-14}$ times smaller than a FDTD cell can be accurately modeled using the standard averaging scheme. ${ }^{4}$ The actual displacements due to the acoustic wave are many orders of magnitude larger than this smallest displacement that can be modeled. So it is relatively easy to model the displacements.

The surface displacements are calculated from the waves recorded at the observation points to demonstrate the effectiveness of the beamforming array. Figure 6(a) is a graph of the actual displacement and the displacement calculated without beamforming for several values of $h$. Here the displacements are obtained by comparing the phase between the data obtained with and without the displacement in the model. The actual displacement of the surface is a differentiated Gaussian pulse with the maximum amplitude of $1 \mu m$. When the observation points are located at $h=1 \mathrm{~cm}$ from the surface, the calculated displacement agrees well with the actual displacement. It is seen that when the observation points are relatively far $(h>5 \mathrm{~cm})$ from the boundary, the displacement obtained from the 


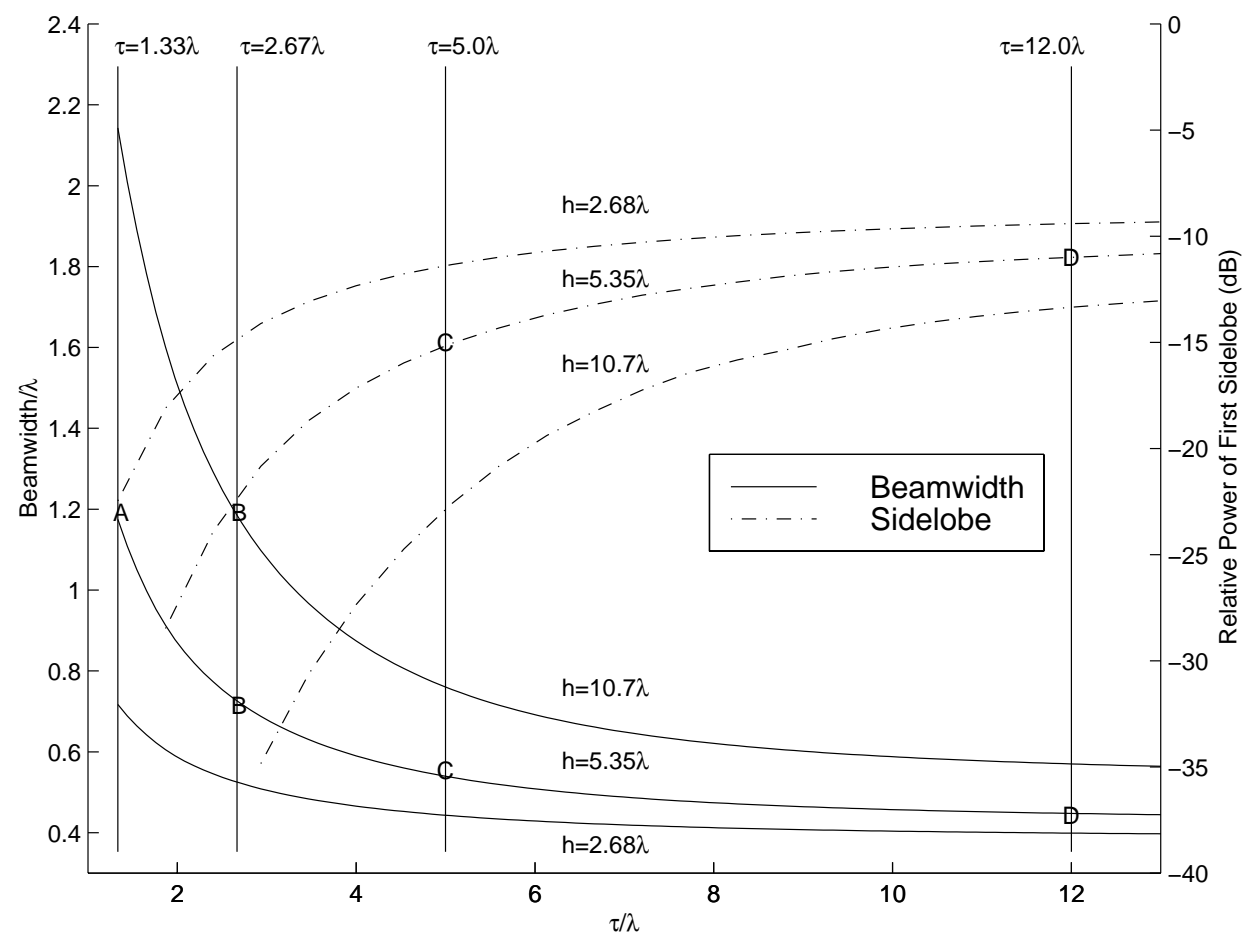

(a)

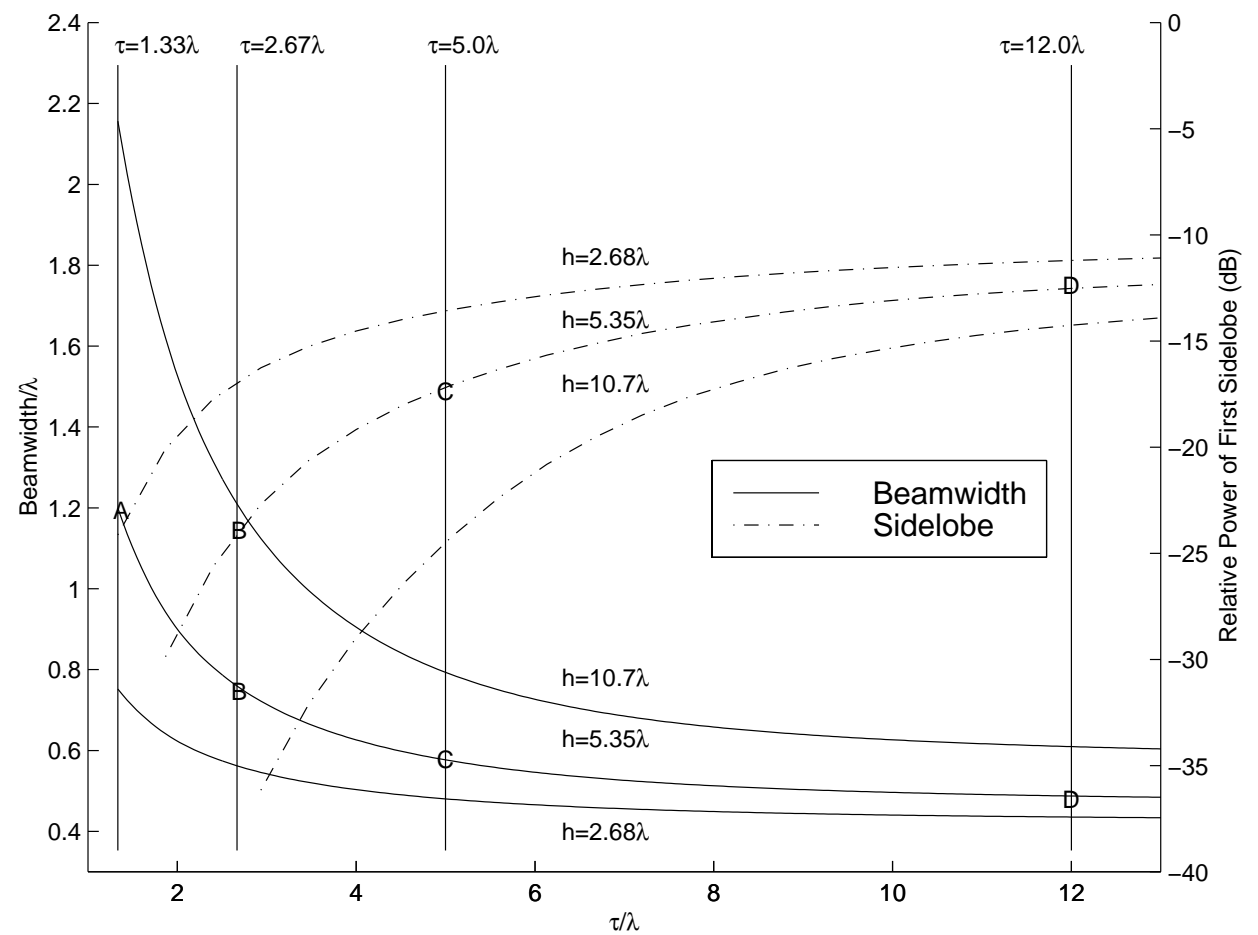

(b)

Figure 3. The beamwidth and the first sidelobe level versus $\tau$ : (a) 2-D Case (b) 3-D Case 


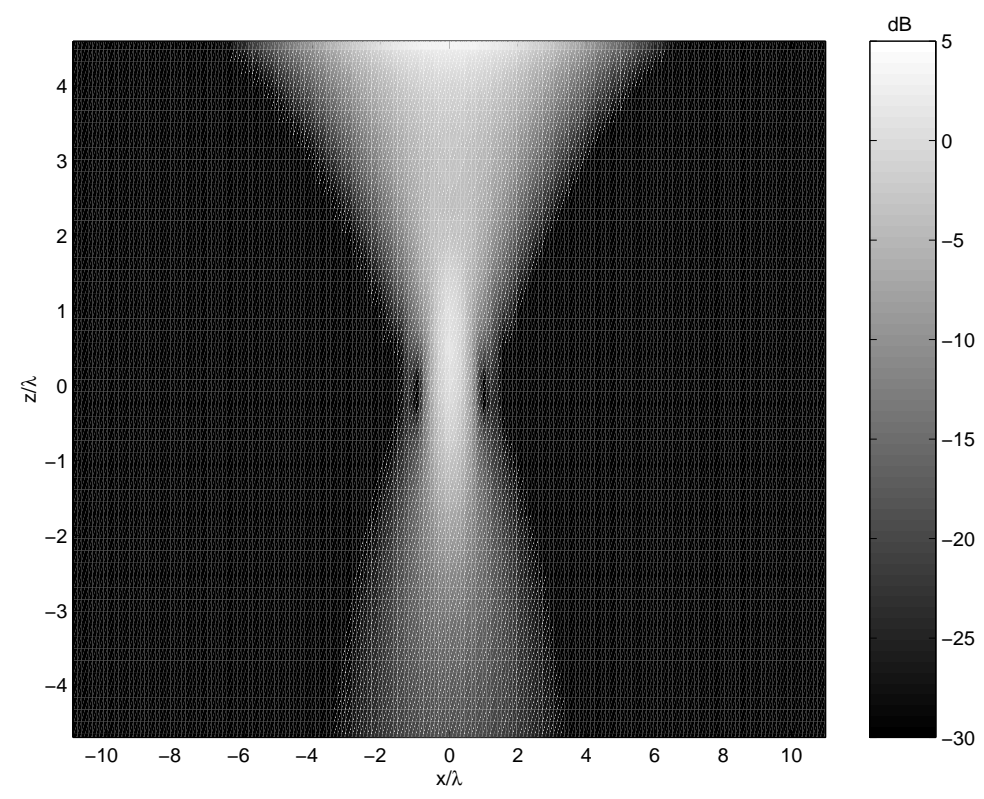

Figure 4. The output of the array when $h=5.33 \lambda$ and $\tau=2.67 \lambda$

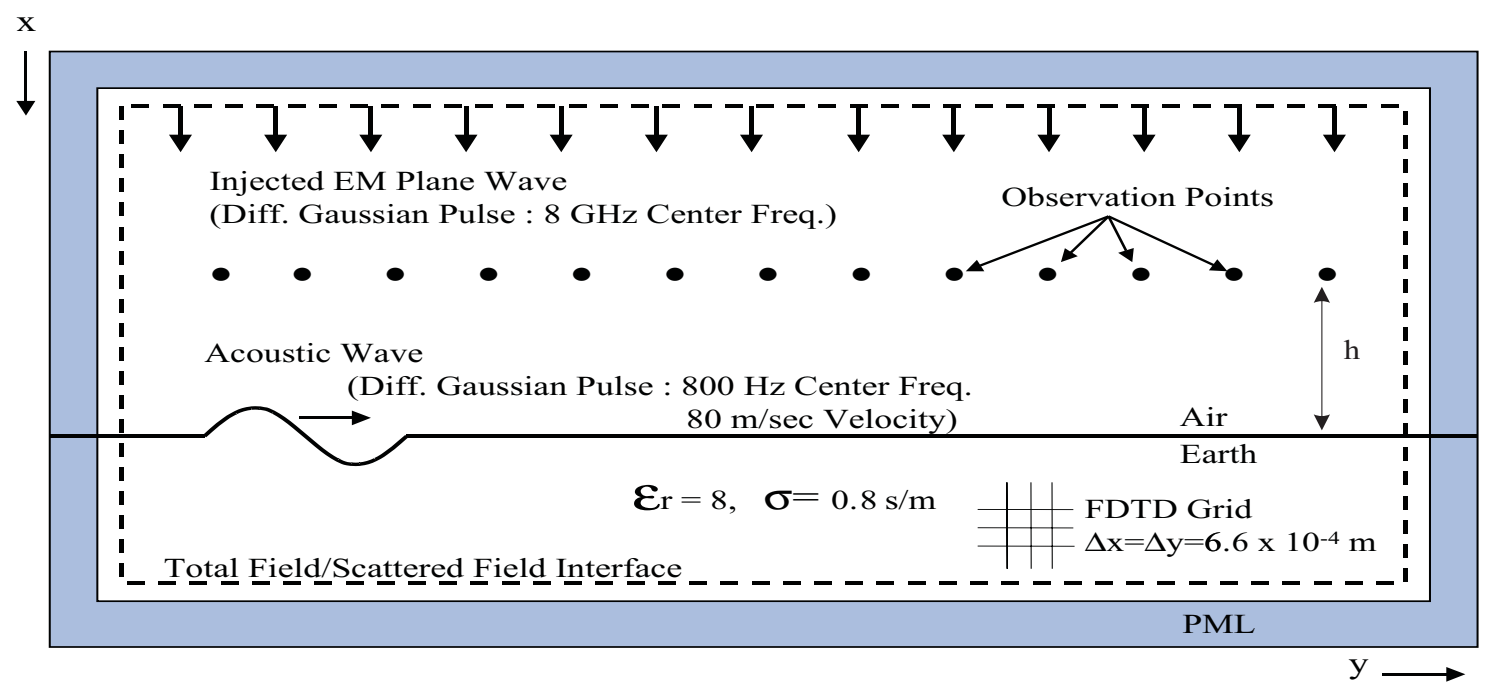

Figure 5. Geometry for FDTD model being investigated

phase at the observation points is not a good replica of the actual displacement of the surface. In order to solve this problem, the beamforming array technique was used to obtain the good image of the displacements.

Figure 6(b) is a graph of the actual displacement and the displacement calculated with beamforming at $h=20 \mathrm{~cm}$ for several values of $\tau$. The pulses in the reconstructed displacements are seen to be somewhat wider than those in the actual displacement. The pulse is seen to be only slightly wider when $\tau=12.0 \lambda$ and is seen to be much wider when $\tau=1.33 \lambda$. This can be explained using Fig.3(a), and the points A,B,C,D on the graph that correspond to the values of $\tau$ and $h$ used in this example. The beamwidth for $\tau=12.0 \lambda$ is seen to be relatively small and is seen to increase with decreasing values of $\tau$. Thus, the increased width of the pulse is seen to be due to the increased beamwidth of the array. A ripple is seen on the reconstructed displacements. The amplitude of the ripple is seen to increase with increasing $\tau$. This is due to the sidelobe levels increasing with increasing $\tau$ as shown in Fig.3(a). 


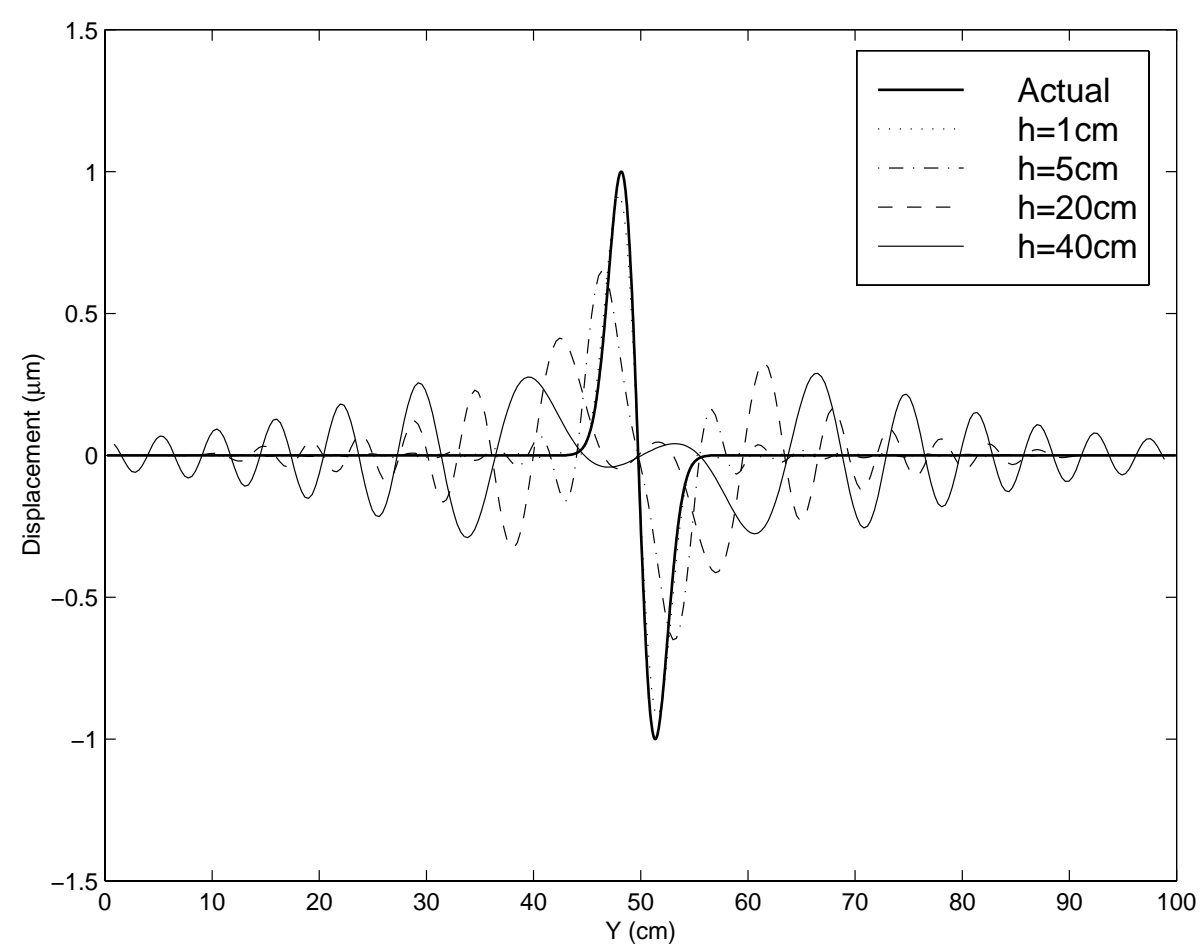

(a)

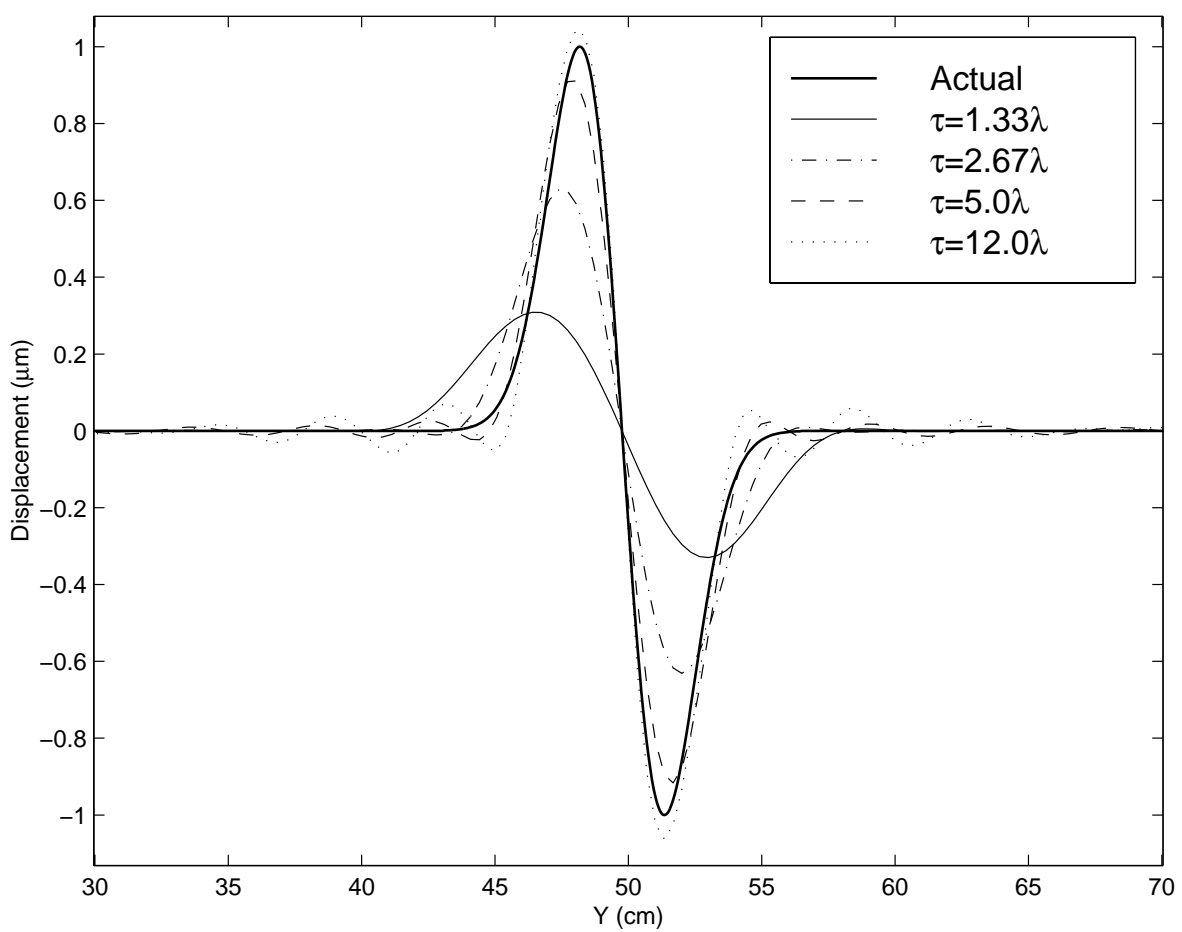

(b)

Figure 6. Actual and computed boundary displacements : (a) Without beamforming, (b)With beamforming when $h=20 \mathrm{~cm}$ 


\section{EXPERIMENTAL RESULTS}

A schematic diagram of the experimental model is shown in Fig.7. It consists of a sandbox and a radar which is scanned over the surface of the sand to implement a synthetic beamforming array. To beamform this array, the phase term in Eq.(2) is doubled to account for the two way travel time of the wave.

The radar is monostatic and uses an open-ended waveguide as the antenna. A circulator is used to help isolate the transmitted and received signals. A HP8720D network analyzer is used to measure the magnitude and phase of the ratio of the received to transmitted signal. The antenna was placed $5.33 \lambda$ above the surface of the sand and the radar was operated at a frequency of $8 \mathrm{GHz}$. The radar is scanned using a $\mathrm{x}-\mathrm{y}$ positioner. The response of the radar is measured on a grid of $59 \times 59$ equally spaced points that are spaced $0.47 \lambda$ apart. The scanned region is approximately $1 \mathrm{~m}$ square. In the sand below the scanned region, one large and two smaller symbols are dug into the sand. A photograph of the symbols is shown in Fig.8(a). Both the large symbol and the smaller symbols are $0.16 \lambda$ high.

The reconstructed height of the surface of the sand calculated from the raw measured data without beamforming is shown in Fig.8(b). The symbols are seen to be very blurred. The reconstructed height of the surface of the sand calculated using the beamforming is shown in Fig.8(c)-(f) for four values of $\tau$. The results with $\tau=1.33 \lambda, 2.67 \lambda$ and $5.0 \lambda$ are seen to be clearly better than the result without beamforming. Note that the resolution of the images increases with increasing value of $\tau$ because of the decreasing beamwidth of the array. However, note that the noise in the image increases with increasing values of $\tau$ because of the increasing sidelobes of the array. The image with $\tau=12.0 \lambda$ is seen to be very noisy. This is predicted by the results in Fig.3. The sidelobes are clearly more important for the 3 -D experimental results than for the 2-D numerical results.

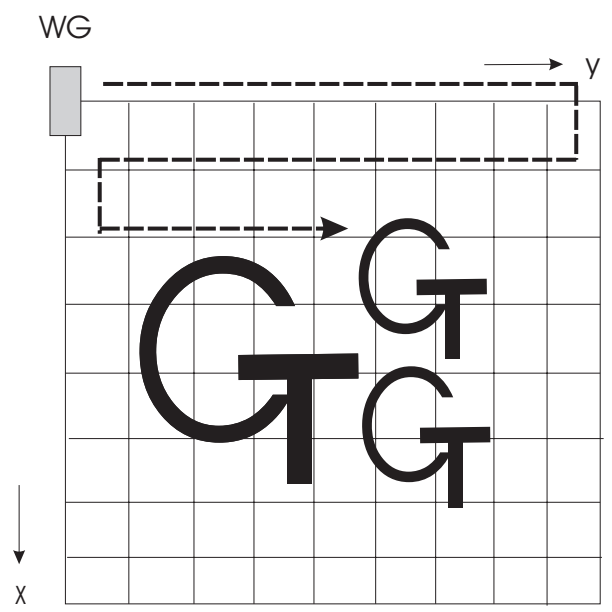

Figure 7. Grid of the region scanned

\section{CONCLUSION}

The feasibility of the beamforming array has been investigated using the FDTD method and the experimental model. By using the beamforming array, very small displacements could be detected in the numerical model, and the surface of the ground was reconstructed in the experimental model. Future plans include extending the experimental model to include time-varying displacements so it can be incorporated into the mine detection system.

\section{ACKNOWLEDGMENTS}

This work is supported in part under the OSD MURI program by the US Army Research Office under contract DAAH04-96-1-0048. 


\section{REFERENCES}

1. W. R. Scott, Jr., C. Schroeder, and J. Martin, "An acousto-electromagnetic sensor for locating land mines," SPIE, AeroSense, Detection and Remediation Technologies for Mines and Minelike Targets III, pp. 176-186, April 1998.

2. S. Gedney, "An anisotropic perfectly matched layer-absorbing medium for the truncation of the fdtd lattices," IEEE Trans. on Antennas and Propagation 44, pp. 1630-1639, 1996.

3. A. Taflove, Computational Electrodynamics:The Finite-Difference Time-Domain Method, Artech House, Boston.London, 1995.

4. S. H. Lee and W. R. Scott, Jr., "Modeling vibrating boundaries with the finite-difference time-domain method," Proc. of the 1998 URSI Radio Science Meeting, p. 70, June 1998. 


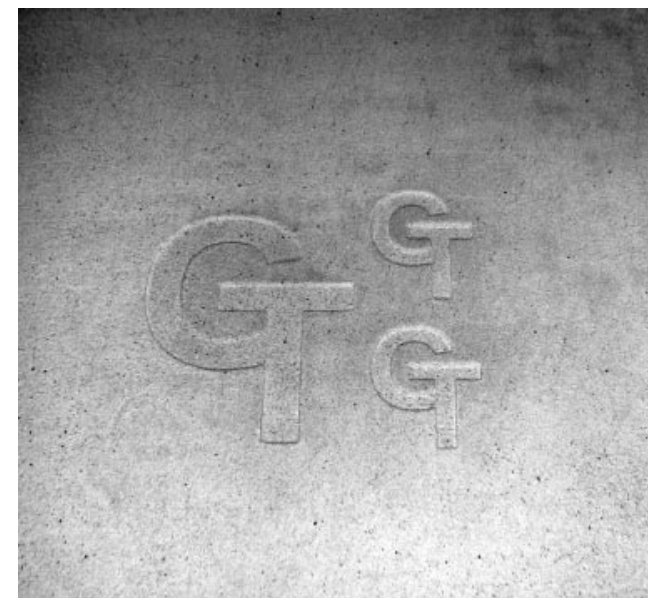

(a)
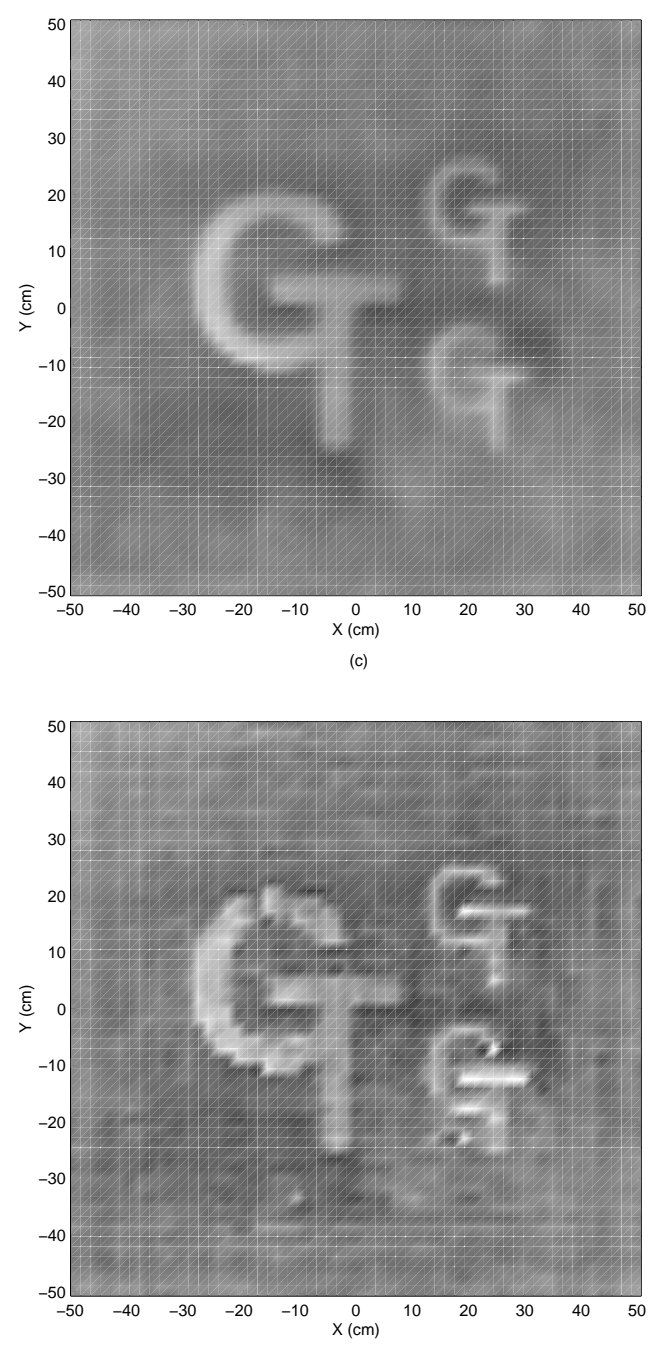
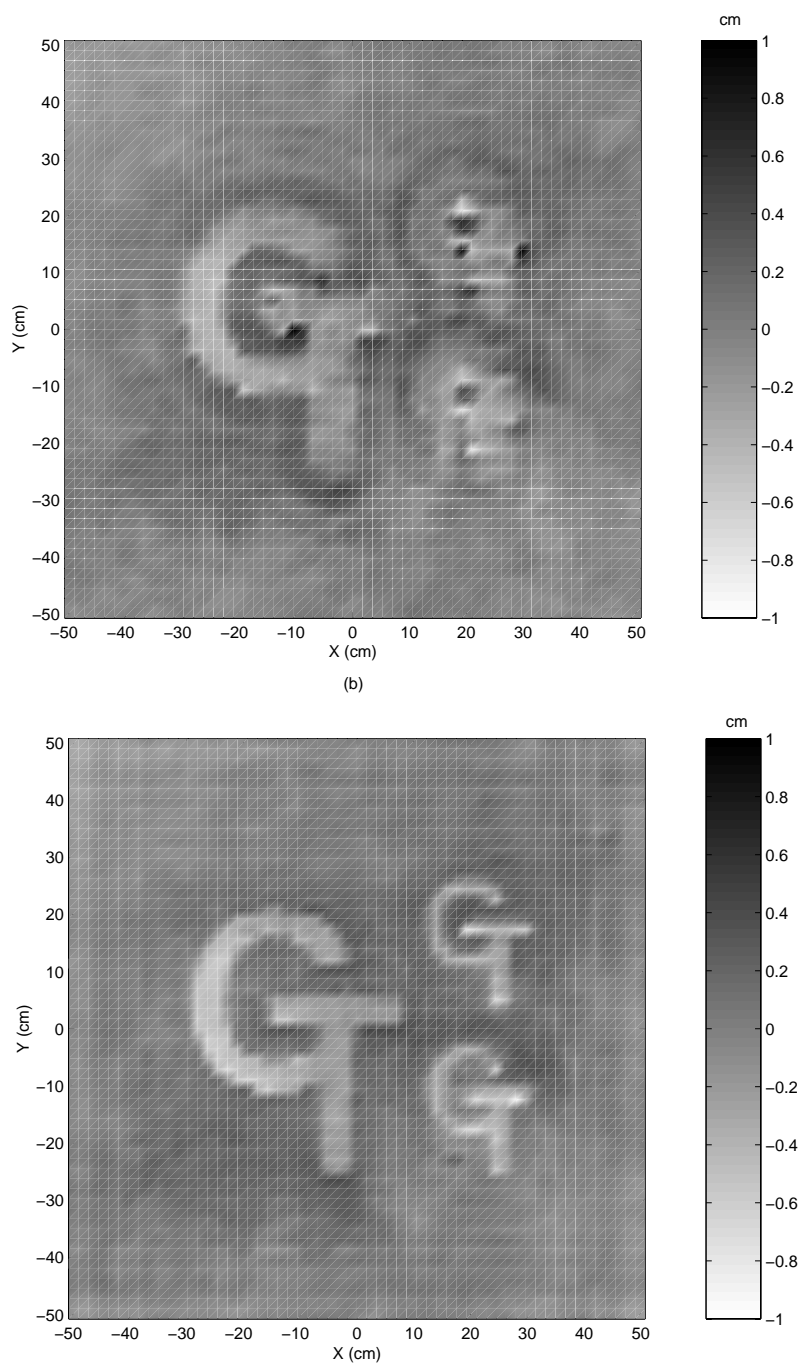

(d)
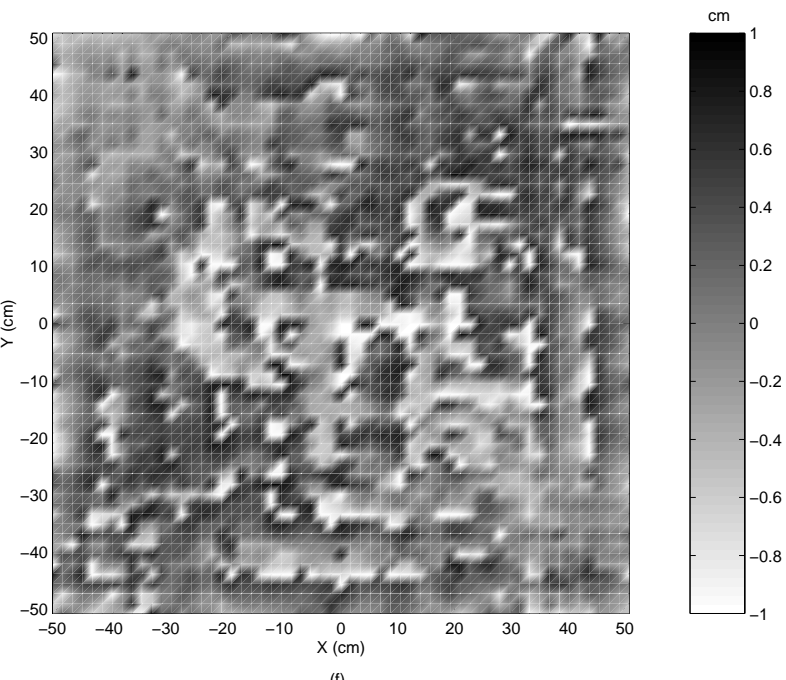

Figure 8. Reconstructed surface of the sand when $\mathrm{h}=20 \mathrm{~cm}$ : (a) Photograph of the surface of the sand, (b) Raw, (c) $\tau=1.33 \lambda$, (d) $\tau=2.67 \lambda$, (e) $\tau=5.0 \lambda$, (f) $\tau=12.0 \lambda$ 